\title{
Additional Host Plants of the Carpenter Bee, Xylocopa brasilianorum (L.) (Hymenoptera: Apoidea), in Puerto Rico'
}

\author{
George C. Jackson ${ }^{2}$
}

ABSTRACT

Ten years of observing the food and nesting habits of the carpenter bee or "cigarrón", Xylocopa brasilianorum (L.), has resulted in the listing of an additional 89 new records of plant species whose flowers provide food for this insect in the form of nectar and pollen. Four additional species were observed as being used as nesting sites, and are first reports for Puerto Rico. It is suggested that the carpenter bee, in her food harvesting activities, may be responsible for the pollination of 18 fruit and seed crops that are used as food sources by man in Puerto Rico.

\section{INTRODUCTION}

The purpose of this paper is to present continued observations concerning the carpenter bee, or "cigarrón", Xylocopa brasilianorum (L.), in Puerto Rico, and is a continuation of the host plant list published in 1976 (8). It is hoped that this report on the additional host plants which provide adult food for this insect will contribute to the knowledge of our single representative of this very widespread genus.

The female carpenter bee in her food harvesting activities may be responsible for the pollination of a large number of local plant species $(2,7,8,13)$. In her foraging, she also visits flowers that have tubular corollas, which may restrict the entrance of insect or avian visitors. In flowers of this type, only nectar is harvested. This is accomplished by perforating the corolla near the calyx by the carpenter bee, or other visitors, such as honey bees, wasps and hummingbirds. This harvesting has been called flower burglary, mutilation, perforation, raiding, robbery, or thievery, depending on the author (3).

\section{MATERIALS AND METHODS}

The female carpenter bee was observed during all seasons of the year, and in practically all of our insular geoclimatological regions. Her food collecting activity in Puerto Rico is diurnal and is apparently controlled by the nectar flow of the particular plant species being visited. Carpenter bees are most abundant and active on bright sunny days. Some venture forth on cloudy days, but thus far, none have been observed collecting food during wet or rainy days.

\footnotetext{
${ }^{1}$ Manuscript submitted to Editorial Board, December 9, 1985.

${ }^{2}$ Former Associate Horticulturist, Agricultural Experiment Station, Mayagüez Campus, University of Puerto Rico, Rio Piedras, P.R. Present address: Caribbean Research Associates, Inc., Romanach 306, Rio Piedras, PR 00926.
} 
Males and females are identified by color: the females are shiny black and the males are a dull yellow. No males were observed collecting food. Since 1964, males have been observed only twice outside the nest. The first instance was a lone male flying in a disoriented pattern, about twelve inches over recently plowed ground at Isabela; and the second, hovering almost motionless outside the nest entrance in a fence post of an undetermined species, near Guavate.

Flowering plants that are food sources for this insect have been noted as to family, genera, species, and geographical location, only after ascertaining that food collection was made. Local accepted common English and Spanish names $(10,15)$ have been included. When a local common name is not published, or is not known, the number in parentheses following a common name indicates its source. Also, numbers in parentheses following Latin plant names indicate reports of Xylocopa spp. other than X. brasilianorum (L.) as being food collectors of that particular plant species, but in other parts of the world. Because of the recent changes in the taxonomic and phytogenetic concepts, the Monocotyledons are placed after the Dicotyledons, as they are now considered to be more modern than the Dicotyledons (10).

\section{HOST PLANTS}

PROTEACEAE

Grevillea robusta A. Cunn.

Silk oak, Roble de seda; at Mayagüez.

POLYGONACEAE

Polygonum punctatum Ell.

(Persicaria punctata (Ell.) Small), Smart weed, Yerba de hicotea; at Caguas.

\section{AMARANTHACEAE}

Amaranthus spinosus L.

Spiny amaranth, Blero espinoso; near Jauca.

LAURACEAE

Licaria triandra (Sw.) Koesterm.

(Misanteca triandra (Sw.) Mez.), Lebisa, Palo misanteco; near Florida.

\section{PAPAVERACEAE}

Argemone mexicana L.

Mexican poppy, Cardo santo; at Coamo. 


\section{CRUCIFERAE}

\section{Lepidium virginicum $L$.}

Wild pepper-grass, Mastuerzo; at Rio Piedras.

\section{CRASSULACEAE}

Echeveria secunda W. B. Booth

(Cotyledon secunda Baker), Ice plant (19); at Isabela.

ROSACEAE

Eriobotrýa japonica (Thunb.) Lindl.

Loquat, Níspero de España; at Mayagüez.

LEGUMINOSAE: MIMOSOIDOIDEAE

Leucaena leucocephala(Lam.) DeWit

(L. glauca (L.) Benth.), Wild tamarind, Campeche; at Lajas.

LEGUMINOSAE: CAESALPINIOIDEAE

Cassia alata $\mathrm{L}$.

(Herpetica alata (L.) Raf.), Ringworm tree, Talantala; at Manatí.

LEGUMINOSAE: PAPILIONOIDEAE

Mucuna deeringiana (Bort.) Merr.

(Stizolobium deeringiana Bort.), Velvet bean, Habichuela terciopelo; at Caguas.

Desmodium tortuosum (Sw.) DC.

(Meibomia purpurea (Miller) Vail), Tick trefoil, Junquillo; at Carolina.

\section{OXALIDACEAE}

Averrhoa carambola L.

Carambola, Jalea; at Punto Cubano.

\section{ZYGOPHYLLACEAE}

Tribulus cistoides L.

Large yellow caltrop, Abrojo; at Santurce.

RUTACEAE

Citrus sinensis (L.) Osbeck

Sweet orange, Naranja dulce; at Adjuntas.

MELIACEAE

Swietenia mahagoni (L.) Jacq. Mahogany, Caoba; at Guayanilla. 


\section{Drypetes glauca Vahl}

Varital (11, 12), Palo blanco; at Mayagüez.

D. laterifolia (Sw.) Krug \& Urban

Guinea-plum, Cueriduro; at Florida.

Hippomane mancinella $L$.

Manchioneel, Manzanillo; at Dorado.

\section{ANACARDIACEAE}

Mangifera indica L.

Mango, Mango; at Rincón.

Spondias mombin $\mathrm{L}$.

(S. axillaris Roxb.), Yellow mombin, Jobo de puerco, at Arús.

SAPINDACEAE

Blighia sapida $\mathrm{K}$. Koenig

(Cupania sapida (K. Koenig) Voight), Akee, Seso vegetal; at Rio Piedras.

Cardiospermum halicacabum "microcarpum" (HBK) Blume (C. microcarpum HBK.), Baloon vine, Bombilla; at Vega Alta.

Melicoccus bijugatus Jacq.

(Melicocea bijuga L.), Spanish lime, Quenepa; mostly in male trees, at Hacienda Florida, Santa Isabel.

Sapindus saponaria $\mathrm{L}$.

(S. indicus Poir.), Soap-berry, Saponaria; at Aguirre.

BALSAMINACEAE

Impatiens balsamina $\mathrm{L}$.

Balsam, Espuela de galán; at Jayuya.

VITACEAE

Cissus sicyoides L.

Pudding vine, bejuco de caro; at Hatillo.

Vitis vinifera $L$.

Grape, Uva; observed only once, possibly collecting pollen; at Juana Díaz.

\section{ELAEOCARPACEAE}

\section{Mutingia calabura L.}

Panama berry, Capulin; at Mayagüez. 
MALVACEAE

Hibiscus syriacus L. "Colestis"

(H. acerifolius Salisb.), Rose of Sharon, Rosa de altea; at Guaynabo. H. tiliaceus L.

(Pariti tiliaceum (L.) St. Hil.), Sea hibiscus $(11,12)$, Emajagua; at Río Piedras.

Malvaviseus arboreus Cav.

(M. malvaviseus (L.) Britton \& Wilson), Sleeping hibiscus, Capucha de monje, at Mayagüez. The carpenter bee perforates the corolla near the calyx, as do wasps and humming birds. Honey bees also gather nectar from this flower, but only after it has been punctured by a previous visitor.

Sida acuta Burm.f.

(S. carpinifolia L.f.), Wire weed, Escoba blanca; at Guayama.

Urena lobata L. ssp. lobata

(U. trilobata Vell.), Bur, Cadillo; at Santa Isabel.

BOMBACACEAE

Adansonia digitata $L$.

Boabab, Boabab; at Aguirre.

Ceiba pentandra (L.) Gaertn.

(C. casearia Medic), Silk-cotton tree, Ceiba; at Hacienda Florida, Santa Isabel.

STERCULIACEAE

Guazuma ulmifolia Lam.

(G. guazuma (L.) Cockerell), West Indies elm, Guácima; at Ponce.

BIXACEAE

Bixa orellana L.

Anatto, Achiote; at Cubuy.

\section{PASSIFLORACEAE}

Passiflora edulis Sims $(16,17)$.

Water lemon fruit, Parcha; at Guayama.

CARICACEAE

Carica papaya $L$.

(C. portoricensis (Solms-Laub.) Urban), Papaya, Lechosa, mostly on male flowers, at Isabela. 


\section{LYTHRACEAE}

Lagerstroemia indica L.

(L. elegans Wallich \& Paxt.), Crepe myrtle, Astromelia; at Aguadilla. COMBRETACEAE

Bucida buceras $\mathrm{L}$.

Black olive, Ucar; at Tallaboa.

Conocarpus erectus $\mathrm{L}$.

Button-tree, Botoncillo; at Río Grande.

MYRTACEAE

Eugenia uniflora $L$.

(E. michelli Lam.), Surinam cherry, Pitanga; at Mayagüez.

Zyzygium malaccense (L.) Merr. \& Perry

(Syzygium cumini (L.) Skeels), Java plum (9), Manzana malaya (9);

at Mayagüez.

PLUMBAGINACEAE

Plumbago auriculata Lam. $(3,17)$.

(P. capensis Thunb.), Blue plumbago, Isabel Segunda; at Aguadilla.

\section{Oleaceae}

Ligustrum japonicum Thunb.

(L. kellermanni Van Houte), Japanese privet, Privet japonesa; at

Rio Piedras.

\section{APOCYNACEAE}

Thevetia peruviana (Pers.) K. Shum. (3).

(Cerbera thevetia L.), Lucky-nut, Cabalonga; at Río Piedras.

\section{ASCLEPIADACEAE}

Cryptostegia grandiflora $\mathrm{R}$. Br.

Purple allamanda, Alamanda morada falsa; at Aguirre.

CONVOLVULACEAE

Ipomoea horsfalliae W. Hooker

Red Princess (20), Campanitas de coral; at Aguas Buenas.

I. nil (L.) Roth

Japanese morning glory (1), Bejuco de vaca; at Vega Baja.

I. purpurea (L.) Roth

Common morning glory (1), Aurora (20); at Rio Piedras.

Merremia tuberosa (L.) Rendle 
(Operculina tuberosa (L.) Meissn.), Wood-rose, Batatilla ventruda; at Aguirre.

BORAGINACEAE

Cordia sebestena $\mathrm{L}$.

(Sebesten sebestena (L.) Britton), Geiger tree, Anaconda; at Salinas. Tournefortia gnaphalodes (L.) R. Br.

(Mallotonia gnaphalodes (L.) Britton), Bay-lavender, Nigua de playa; at Dorado.

VERBENACEAE

Avicenna germinans (L.) L.

(A. nitida Jacq.), Black-mangrove (11, 12), Mangle prieto (11, 12); at Luquillo beach.

Petrea volubilis L.

Purple wreath, Petrea; at Arecibo.

$$
\text { SOLANACEAE }
$$

Petunia hybrids

Petunia, Petunia; at Caguas.

\section{SCROPHULARIACEAE}

\section{Bacopa monnieri (L.) Pennell}

(Bramia monnieri (L.) Drake), Water hyssop (1), Yerba de culebra; at Guánica.

Stemodia durantifolia (L.) Sw.

Verónica; at Rio Piedras.

\section{BIGNONIACEAE}

Pyrostegia venusta (Ker-Gawl.) Miers

(P. ignea (Vahl) K. Presl), Flame vine, Flor de Venus; at Rio Piedras. The carpenter bee harvests nectar after puncturing the corolla near the calyx; honey bees also visit, but only after the flower has been perforated by the carpenter bee.

Saritaea magnifica (Sprague) Dugand

(Arrabidaea magnifica Sprague), Purple begonia (20); at Trujillo Alto. The carpenter bee perforates the corolla near the calyx and collects nectar.

Tabebuia rosea (Bertol.) DC. (6).

(T. pentaphylla Bertol.), Pink trumpet-tree (12), Roble venezolano; at Cayey. The carpenter bee harvests nectar through a perforation she makes in the corolla near the calyx. Honey bees also harvest nectar in this manner. 
RUBIACEAF,

Coffea arabica $L$.

Coffee, café; at Las Marias.

Hamelia patens Jacq.

(H. erecta Jacq.), Scarletbush (11), Bálsamo; at Corozal.

Mussaenda erythrophylla Schuhmach. \& Thonn.

Scarlet mussaenda, Colombina roja; at Rio Piedras. Humming birds also visit and harvest nectar from the small corolla.

M. philippica A. Rich.

Colombine, Colombina; at Rio Piedras.

Mussaenda hybrid "Doña Aurora".

Double white hybrid; at Rio Piedras.

Pentas lanceolata (Forssk.) Deflers

(Ophiorhiza lanceolata Forssk.), Egyptian starcluster, Pentas; at Adjuntas.

Richardia scabra L.

(Richardsonia scabra (L.) St. Hil.), Mexican clover (5), Verdolaga de Florida (4); at Isabela.

\section{CAPRIFOLIACEAE}

Lonicera japonica Thunb. (3).

Honeysuckle, Madreselva; at Aguirre. The carpenter bee and honey bees harvest nectar through the basal puncture in the corolla, close to the calyx.

\section{CUCURBITACEAE}

Citrulus lanatus (Thumb.) Matasumura \& Nakai (16).

(C. citrullus (L.) Karst.), Watermelon, Sandia; Santa Isabel.

Momordica charantia L.

Wild balsam apple, Cundeamor; at Ponce.

Sechium edule (Jacq.) Sw.

Christophine, Chayote; at Utuado.

\section{COMPOSITAE}

Bidens alba (L.) DC. "radiata" (Sch.-Bip.) Ballard

(B. pilosa L.), Shepherd's needles, Margarita; at Guayama.

Mikania cordifolia (L.f.) Willd.

Guaco; at Quebradillas.

Parthenium hysterophorus L.

Mugwort, Artemisa cimarrona; at Rio Piedras.

Vernonia cinerea (L.) Less.

(Conyza cinerera L.), Ironweed (1), Yerba socialista; at Yauco. 
Wedelia reticulata $D C$.

Manzanilla del monte; at Aguadilla.

\section{ALISMATACEAE}

Sagittaria lancifolia $\mathrm{L}$.

Arrow leaf, Flecha de agua; at Santurce.

PALMACEAE

Arecastrum romanzoffianum (Chamisso) Beccari

(Cocos romanzoffianum Cham.), Queen palm (1), Cocos plumoso; at Río Piedras.

Corypha umbraculifera $\mathrm{L}$.

Talipot palm, Palma talipot; at Mayagüez. This species bloomed at TARS during 1982, and in and around the gigantic inflorescence, numerous carpenter bees, honey bees, wasps and assorted Diptera were observed harvesting nectar or pollen. This same activity of an unknown Xylocopa spp. was also noted by the author in a flowering specimen at main entrance of the Bernard Lodge Estates, located in Saint Catherine's Parish, Jamaica, in June, 1984.

PONTEDERIACEAE

Eichhornia crassipes (Mart.) Solms-Laub. (Piaropus crassipes (Mart.) Britton), Water hyacinth, Jacinto de agua; at the Coamo Dam.

\section{LILIACEAE}

Dracaena fragrans (L.) Ker-Gawl.

Indian cane, Dracina; at Río Piedras.

Yucca aloifolia L.

Spanish bayonet, Bayoneta española; at Río Piedras.

\section{ZINGIBERACEAE}

Costus indica (Koenig) J. E. Smith

Crepe ginger (1); at Rio Piedras.

CANNACEAE

Canna indica $\mathrm{L}$.

(C. coccinea Miller), Wild arrowroot, Maraca; at Trujillo Alto.

ORCHIDACEAE

Arundina graminifolia (D. Don) Hochr.

(A. bambusifolia Lindl.), Bamboo orchid (1); at Guaynabo. 
Dendrobium hybrids.

Known as the reed-stem dendrobiums; at Guaynabo.

\section{NESTING SITES}

The female carpenter bee in her nesting activities utilizes a number of plants or plant derivatives $(8,14,18,21)$. To the previous listed thirteen species of wood used, the following four are added. In the Rio Piedras area, the carpenter bee also nests in the dead-wood of lime, Citrus aurantifolia Sw.; Santa Maria, Calophyllum brasiliense Jacq.; the Queen of flowers, Lagerstroemia speciosa (L.) Pers., and in untreated construction pine timbers, Pinus spp.

\section{DISCUSSION}

The 89 listed food plant species belonging to 82 genera and 51 families represent a fairly large segment of our insular flora, and they are first records for Puerto Rico. The fruit or seeds of 18 or more of these reported plants, such as akee, anatto, balsam apple, carambola, christophine, coffee, grape, Java plum, loquat, mango, Panama berry, papaya, Spanish lime, Surinam cherry, sweet orange, water lemon fruit, watermelon, and yellow mombin are utilized as human food sources in the tropics. It should not be overlooked, that the carpenter bee, in the process of food gathering, may inadvertently be directly or indirectly responsible for their pollination.

This host plant list, combined with the list published in 1976 (8), makes a total of 374 species of food host plants of this insect. Of this number, the fruits or seeds of at least 43 are food sources of mankind. The addition of four new nesting sites makes a total of 17 woody species used for this purpose in Puerto Rico.

\section{RESUMEN}

Notas acumuladas durante 10 años de observaciones informan por primera vez en Puerto Rico que el cigarrón aprovecha como fuente de alimento unas 89 especies de plantas adicionales a las que se informaron en 1976. El cigarrón anida en cuatro especies leñosas, que también se informan por primera vez en Puerto Rico. En estas plantas, en cuyas flores el cigarrón o "carpenter bee", Xylocopa brasilianorum (L.), encuentra alimento (néctar y polen), también encuentra en algunos casos madera para sus nidos. Se sugiere que el cigarrón, inadvertida, directa o indirectamente, es responsable de la polinización de 18 o más especies adicionales de frutas y semillas que constituyen una fuente de alimento para el nombre. 


\section{LITERATURE CITED}

1. Bailey, L. H. and Ethel Zoe Bailey, 1976. Hortus Third-a concise dictionary of plants cultivated in the United States and Canada, MacMillan Publ. Co., New York, illus. $1290 \mathrm{pp}$.

2. Barnés, D. K. and R. H. Freyre, 1969. Seed production potential of Tephrosia vogelii in Puerto Rico. J. Agric, Univ. P.R., 53 (2): 207-12.

3. Barrows, E. M., 1980. Rolubing of exotic plants by introduced carpenter and honey bees in Hawaii, with comparative notes. Biolropica, 12 (1): 23-29.

4. Blázquez, C. H. and M. Rodriguez de la Torre, 1978. Plantas nocivas y cómo combatirlas, Vol. II, Editorial Lamusa, México, 575 pp.

5. Cárdenas, J., C. E. Reyes and J. D. Doll, 1972. 'Tropical weeds-Malezas tropicales, I. C. A., Bogatá, illus. 341 pp.

6. Gentry, A. H., 1974. Coevolutionary patterns in Central American Bignoniaceae. Ann. Mo. Bot. Gard., 66: 728-52.

7. Jackson, G. C. and J. Bauzá Salas, 1965. Insect visitors of Lecythis elliptica HBK. J. Agric. Univ. P.R., 49 (1): 133-41.

8. — - and R. O. Woodbury, 1976. Host plants of the carpenter bee, Xylocopa brasilianorum (L.) (Hymenoptera : Apoidea) in Puerto Rico. J. Agric. Univ. P.R., 60 (4): 639 60.

9. Kennard, W. C. and H. F. Winters, 1960. Some fruits and nuts for the tropics. USDA Misc. Publ. 801, illus. 135 pp.

10. Liogier, H. A. and L. F. Martorell, 1982. Flora of Puerto Rico and adjacent islands: a systematic synopsis. Editorial Univ P.R., Río Piedras, $343 \mathrm{pp}$.

11. Little, L. Jr. and F. H. Wadsworth, 1964. Common trees of Puerto Rico and the Virgin Islands, USDA Agric. Handb. 249 , illus, 548 pp.

12. - R. O. Woodbury and F. H. Wadsworth, 1974. Trees of Puerto Rico and the Virgin Islands, USDA Agric. Handb. 449, illus., 1024 pp.

13. Martin, F. W., E. Cabanillas and S. Ortiz, 1963. Natural pollination, hand pollination and crossability of some Mexican species of Dioscorea. Trop. Agric. Trin. 40 (2): 13541.

14. Martorell, L. F., 1945. A survey of the forest insects of Puerto Rico: Part II. J. Agric. Univ, P.R., 29 (4): 564.

15. - H. A. Liogier and R. O. Woodbury, 1981. Catálogo de los nombres vulgares y cientílicos de las plantas de Puerto Rico, Esta. Exp. Agric. Univ. P.R. Bol. 263, 231 pp.

16. Nishida, T., 1958. Pollination of passion fruit in Hawaii. J. Econ. Ent., 51 (2): 146-56.

17. — 1963. Ecology of the pollinators of passion fruit. Hawaii Agric. Exp. Stn. Tech. Bul, 55 , illus., $38 \mathrm{pp}$.

18. Ranos, J. A., 1946. The insects of Mona Island (West Indies), J. Agric. Univ. P.R., 30 (1): 70 .

19. Robertson, E. T. and E. G. B. Gooding, 1963. Botany in the Caribbean, Collins Press, London, illus., $246 \mathrm{pp}$.

20. Steiner, Mona Lisa, 1960. Philippine ornamental plants, and their care. McCullough, Manila, illus., $233 \mathrm{pp}$.

21. Wolcolt, G. N., 1948. Insects of Puerto Rico, J. Agric. Univ. P.R., 33 (4): 871-72. 